Seyla Benhabib*

\title{
Habermas' neue Phänomenologie des Geistes: Zwei Jahrhunderte nach Hegel
}

https://doi.org/10.1515/dzph-2021-0046

\begin{abstract}
Jürgen Habermas's opus magnum, Auch eine Geschichte der Philosophie, synthesises his impressive work of the last half century. His thesis is that the modern project of the normativity of "rational freedom" can be reconstructed as a learning process of the conflictual dialogue between reason and faith, philosophy and religion in the West. Furthermore, under conditions of a world society, cross-cultural communication across lifeworlds, based on such normative principles, is possible.

I argue that Habermas's argument recapitulates a claim first made in The Phenomenology of Spirit by Hegel, who presented the normativity of modernity through a narrative unfolding between two epistemological standpoints, namely, that of consciousness and "we." Just like Hegel, in order to defend the idea of a Lernprozess, Habermas too must presuppose a unified subject called "we;" furthermore the development of such subjectivity unfolds in a homogeneous temporal process that is then assumed to be the same for all mankind.

I call this a form of "historicism," and juxtapose recent historical writing that presents the narrative of modernity and the emergence of world-society as a much more diverse and fractured process than Hegel's and Habermas's methodology. "Die Einbeziehung der Anderen," I argue, must involve including the voices of those others who do not experience the normative of modernity as a process like the one unfolding between faith and reason in the West.

Nevertheless, I conclude that this plea for a more complex narrative that "provincialises Europe," (Dipesh Chakrabarty) is not a rejection of the normative legacy of modern rationality and freedom that are based on the ideals of fallibilism, refutability and revisability through a rational community of inquirers.
\end{abstract}

Keywords: Jürgen Habermas, Auch eine Geschichte der Philosophie, G. W. F. Hegel, Phenomenology of Spirit, normativity of modernity, Dipesh Chakrabarty, provincializing Europe

*Kontakt: Seyla Benhabib, Columbia University, Columbia Law School, Jerome Greene Hall, 435 West 116th Street, New York NY 10027, USA; sb3862@columbia.edu 


\section{Von der Theorie des kommunikativen Handelns zu Auch eine Geschichte der Philosophie}

Jürgen Habermas' opus magnum, Auch eine Geschichte der Philosophie (AGP), ist wie eine dicht geknüpfte, über zwei Flussufer geworfene Seilbrücke, die verschiedene Argumentationsstränge zusammenbringt, die ihn über die Jahre beschäftigt haben. ${ }^{1}$ Die Reisende muss Sorge tragen, die Stärke des Seils und seiner verschiedenen Litzen zu beachten, damit sie auf der Reise durch die Jahrhunderte der Philosophiegeschichte nicht ihren eigenen Weg verliert.

Zwischen der Veröffentlichung der Theorie des kommunikativen Handelns (TKH) und dem fast vierzig Jahre späteren Erscheinen von AGP 2019 hat sich die Weltgesellschaft derartig geändert, dass die neomarxistische Neuaneignung von Max Webers Theorie der Moderne als einem Prozess der Entzauberung und Verdinglichung, wie im Werk von György Lukács, nicht mehr angemessen scheint. In der TKH ging es Habermas darum zu zeigen, dass der „Bedeutungs-“ und „Freiheitsverlust“, die Weber als unvermeidliche Folgen der Moderne prognostiziert hatte, nicht eingetreten waren, und dass der Freiheitsverlust, den Webers berühmte Formel vom „stahlharte[n] Gehäuse“ der Moderne prophezeite, überwindbar war. ${ }^{2}$ Freiheit und individuelle Autonomie ließen sich weiterhin bewahren. Doch die Unzufriedenheit mit der Moderne blieb verbreitet und wiederkehrend. Wie Weber bereits vorweggenommen hatte, würde es diejenigen geben, die „dies Schicksal der Zeit nicht männlich ertragen“ konnten, und er wies sie darauf hin, dass die „Arme der alten Kirche“ offen blieben, wie die einer alten Mutter, die darauf wartet, dass ihr launischer Nachwuchs nach Hause zurückkehrt. ${ }^{3}$

Weber hatte sowohl Unrecht als auch Recht: Unrecht in dem Sinne, dass er nicht vorhersah, dass viele Gesellschaften und Kulturen, die keine Säkularisierung und Entzauberung erfahren hatten, alternative Wege in die Moderne nehmen würden, so in China, Japan, Indien und Teilen des Nahen Ostens und Afrikas. In diesen Gesellschaften überleben traditionale und sogar archaische Kulturüberreste bis auf unsere heutigen Tage. Weber hatte dennoch damit Recht, dass westliche wirtschaftliche Modernisierung, Staatserrichtung, öffentliche und

\footnotetext{
1 Bei diesem Beitrag handelt es sich um die von der Autorin selbst editierte deutsche Fassung von: Seyla Benhabib, Habermas' new Phenomenology of Spirit: Two centuries after Hegel, in: Constellations 28.1, 33-44, (C) 2021 John Wiley \& Sons Ltd. Dem Verlag John Wiley and Sons ist für die Genehmigung eines Abdrucks in deutscher Sprache zu danken.

2 Weber (2010), 201. Vgl. ders. (1994), 22; Habermas (1995a), 332-366; Benhabib (1986), 224-278. 3 Weber (1994), 23.
} 
private bürokratische Verwaltung - und heutzutage zunehmend das weltweite Internet und andere soziale Medien der Kommunikation und Information - einen Strudel erzeugen würden, in den alle menschlichen Gemeinschaften, selbst in den fernsten Weltteilen, hineingezogen würden.

Wie können jahrhundertealte kulturelle Lebenswelten und religiöse Traditionen dem Mahlstrom weltweiter Modernisierung widerstehen? Was wird aus menschlichen Gemeinschaften, die zwischen den Imperativen funktionaler Modernisierung auf der einen Seite und den Normen ihrer kulturellen und religiösen Lebenswelten auf der anderen gefangen sind? Um Benjamin Barbers einprägsame Formulierung zu zitieren: Wie können „Dschihad“ und „McWorld“ versöhnt werden $?^{4}$ Da sie den Rahmen des territorial umgrenzten Nationalstaats als selbstverständlich annahm, analysierte die TKH die Widersprüche und Pathologien zwischen System und Lebenswelt ausschließlich in diesem Kontext. Die Weltgesellschaft ${ }^{5}$ wurde nicht thematisiert und alternativen Modernen wurde nicht der ihnen gebührende Platz eingeräumt. ${ }^{6}$

Habermas' Beschäftigung mit den Fragen von Glaube und Vernunft ist zu einem großen Teil seinem Versuch geschuldet, zu ermitteln, ob unter den Bedingungen einer Weltgesellschaft eine interkulturelle Kommunikation über Glaubenswelten hinweg möglich sein würde. ${ }^{7}$ Die gesamte Thematik des Postsäkularismus geht aus diesem Zusammenhang hervor; Postsäkularismus heißt nicht nach dem Säkularismus, sondern jenseits des Säkularismus. Westeuropäische Gesellschaften sind entschieden säkular geblieben; nur im Rest der Welt hat die

4 Barber (1996).

5 „Damit stellt sich die Frage, ob auch die globale Gesellschaft noch in jenen, am westlichen Modell abgelesenen Kategorien gesellschaftlicher Evolution aufgeht, oder ob wir das Standardmodell der hochmobilen und fortschreitend individualisierten, staatlich organisierten und wissenschaftlich rationalisierten Wirtschaftsgesellschaft revidieren müssen, um der kulturellen Variation verschiedener Modernitäten im Rahmen derselben multikulturellen Weltgesellschaft gerecht zu werden“ (Habermas 2019a, 113, Hervorh. im Orig.). Habermas bespricht anschließend verschiedene Antworten, die „systemfunktionalistische“ Analysen gegenüber solchen des „radikalen Kulturalismus“ sowie auf ,vergleichende Zivilisationsforschung“ aufbauenden auf diese Frage geliefert haben (ebd., 113-125).

6 Vgl. Eisenstadt (2000, 2002, 2003).

7 Thomas A. McCarthy, der eine hervorragende Analyse dieser Prozesse vorgelegt hat, paraphrasiert Charles Taylor wie folgt: ,[A]ssuming that some degree of convergence in economic, governmental, and legal institutions and practices is an unavoidable feature of a globalized modernity, what kinds and degrees of divergence remain possible and desirable? In particular, how much room do such modernizing tendencies leave for deep cultural differences?“ (McCarthy, 2009, 159). Vgl. Taylor (2001), 172-176. 
Spätmoderne ein Wiederaufleben der Religion mit sich gebracht, obwohl manche Länder, wie die Vereinigten Staaten, nie gänzlich säkular gewesen sind. ${ }^{8}$

In Auch eine Geschichte der Philosophie verteidigt Habermas Menschenwürde und Rationalität in einem Zeitalter, in dem wir zwischen den Ufern von Naturalismus und Religion gestrandet sind. Diese quaestio ${ }^{9}$ vollzieht sich in Form einer Rekonstruktion der Geschichte der „abendländischen Vernunft“ von der Achsenzeit an über die Kirchenväter, Luther, die wissenschaftliche Revolution, die französischen und schottischen Aufklärungen bis zu Kant, den deutschen Idealisten, und weiter zu Feuerbach, Marx, Kierkegaard und Peirce. Seine Behauptung ist, dass die Begegnung zwischen Glauben und Wissen im Westen einen Lernprozess enthält, der „rational rekonstruiert“ werden kann. Philosophie hebt die Glaubenswahrheiten in einem Prozess auf, den Rainer Forst „rettende Übersetzung“ nennt. ${ }^{10}$ In einer markanten Formulierung schreibt Habermas, Religion bleibe „ein Stachel im Bewusstsein einer säkularen Gesellschaft“11. Die gegenwärtige Konstellation von Philosophie, Naturwissenschaft und Religion muss im Lichte einer „rationalen Rekonstruktion der Entstehungsgeschichte der eigenen Denkweise“ verstanden werden, wie sie am Ende dieses Prozesses erscheint. ${ }^{12}$

Sicherlich waren es nicht nur diese widersprüchlichen Entwicklungen in der Weltgesellschaft, die die Frage der Religion aufs Tapet brachten. Auch in hochgradig säkularen Gesellschaften begannen Fortschritte in Gentechnik und künstlicher Intelligenz Dilemmata aufzuzeigen, auf die weder Naturwissenschaftlerinnen noch Philosophinnen überzeugende Antworten geben konnten. Während die öffentliche Sphäre der Vereinigten Staaten grundsätzlich gefangen in Fragen blieb wie „wann beginnt das Leben“?, „wann ist es moralisch rechtfertigbar, zukünftiges Leben zu beenden?“, fragten andere Gesellschaften: „Sollte die Gesellschaft die Wünsche des Individuums danach, sein Leiden möge beendet und sein Leben geendet werden, respektieren?“; „Wer sollte entscheiden: das Individuum? die Familie? oder die staatliche Bürokratie?“

Habermas folgerte, dass eine postmetaphysische Philosophie, das heißt eine Philosophie, die nicht mehr in den Sicherheiten eines Weltbildes oder eines Bildes des Kosmos gegründet war, keine verbindlichen Antworten auf diese Fragen geben konnte. Stattdessen mussten Individuen sich einem Reich der

\footnotetext{
8 Habermas (2019a), 79.

9 Das Wortspiel „the quest(ion)“ im englischen Original ist nur begrenzt übersetzbar (Anm. d. Übers.).

10 Forst (2021); vgl. Habermas (1979).

11 Ders. (2019a), 86.

12 Ebd., 109.
} 
Bedeutung zuwenden, mit dem die Philosophie immer im Wettbewerb gestanden hatte, nämlich der Religion. Religiöse Traditionen und Gemeinschaften öffneten Herz und Geist von Individuen für einen transzendenten Bereich, der Trost im Angesicht von weltlichem Leiden, Hinfälligkeit, Elend und Tod bot oder ganz einfach im Angesicht der Rätsel des Menschseins. Für Weber lag „Theodizee“, die Rechtfertigung menschlichen Leidens in einer ungerechten und undurchdringlichen Welt, im Herzen aller Weltreligionen. Die beste Antwort, die die Philosophie im postmetaphysischen Zeitalter gegenüber all diesen Fragen bieten konnte, war vielleicht „moralischer Zufall“13 und nicht mehr als das.

In der Zwischenzeit hatten Fortschritte in künstlicher Intelligenz und Robotik, am Gipfel eines Prozesses, der bereits mit der wissenschaftlichen Revolution im 17. Jahrhundert begonnen hatte, sich so weit entwickelt, dass sie uns aller Illusionen beraubten, die wir über unsere besondere Stellung im Kosmos als Menschen gehabt haben mögen. In einem eindrücklichen Beitrag zur Gentechnikdebatte stellte Habermas fest, dass die ontologische Symmetrie unter Generationen von Menschenwesen garantierte, dass ihre Eltern nicht ihre Macher seien ${ }^{14}$; weder Eltern noch ihre Kinder konnten ihren Nachwuchs je nach Gefallen ,produzieren“. Diese Symmetrie müsste aufrechterhalten werden, indem alle Anwendungen der Genmanipulation außer therapeutischen verhindert würden. Kein Elternteil sollte beispielsweise die Mittel haben, ein Kind mit blauen Augen und einem genialen IQ ins Leben zu designen. Das Element genetischer Unvorhersagbarkeit, das mit jedem Geburtsakt einherging, ist eine Vorbedingung der menschlichen Freiheit. Dies erinnert uns an Hannah Arendts Konzept der „Natalität“: Die Geburt jedes Kindes verkündet einen neuen Anfang, da die Handlungen und Worte jedes Kindes sich von jedem anderen unterscheiden, und dies in unvorhersagbarer Weise. ${ }^{15}$ Natalität heißt, dass Kinder keine Produkte ihrer Eltern, sondern ihre eigenen freien Wesen sind. Diese ontologische Symmetrie zwischen Eltern und ihren Nachkommen - dass erstere letztere niemals machen können - ist zentral für unsere Conditio humana. Gefangen zwischen Naturalismus, ${ }^{16}$ der Menschen auf intelligente Maschinen reduzieren würde, die eines Tages von noch intelli-

13 Williams (1984).

14 Vgl. Habermas (2001).

15 Arendt (1967).

16 Nishin Nathwani und Joel Whitebook haben die Frage aufgeworfen, ob „Naturalismus“ der richtige Ausdruck ist, um die Phänomene zu beschreiben, um die es Habermas geht, nämlich reduktionistische Modelle des menschlichen Bewusstseins und Verhaltens, die analog zu intelligenten KI-Systemen aufgebaut sind. Ich stimme diesem Einspruch zu, da „Naturalismus“ überhaupt keinen Reduktionismus implizieren muss. Aber ich werde die Bezeichnung so verwenden, wie es Habermas selbst tut. 
genteren kontrolliert werden könnten, und Religion, die nach Antworten auf die Rätsel und die Misere der menschlichen Existenz suchte - wo stand da die Philosophie? Und was war die angemessene Stelle von Religion in der liberal-demokratischen öffentlichen Sphäre im Hinblick auf die postsäkularen Verhältnisse?

John Rawls kämpfte durch sein ganzes Leben und Werk hindurch mit dieser Frage. ${ }^{17}$ Politischer Liberalismus war, so argumentierte er, nur möglich, wenn Bürgerinnen in derartigen Gesellschaften eine Trennlinie zwischen vernünftigen und unvernünftigen umfassenden Weltsichten ziehen konnten. Vernünftig waren jene, in denen Bürgerinnen einander als gleiche Wesen sahen, denen die Fähigkeit gegeben war, Auffassungen des Guten zu bilden und sie innerhalb eines kooperativen Gerechtigkeitsrahmens zu verfolgen. In der öffentlichen Sphäre liberal-demokratischer Gesellschaften waren Bürgerinnen zudem frei, Argumente auf Grundlage ihrer umfassenden Weltsichten einzuführen, falls und insoweit diese Argumente auch kompatibel mit den Einschränkungen der öffentlichen Vernunft waren. ${ }^{18}$

Es ist unnötig, hier die Rawls-Habermas-Debatte zu rekapitulieren. ${ }^{19}$ Die Diskussion von Rawls in AGP schlägt jedoch einen neuen Ton an. Habermas kritisiert Rawls’ Das Recht der Völker (1999) dafür, dass er illiberale Gesellschaften in achtbare hierarchische, belastete und Schurkenstaaten einteilt. Diese Klassifikation, so Habermas, spiegelt den strategischen Standpunkt aufgeklärter „Global Player“20 wider. Er beharrt weiter darauf, dass mit Blick auf die heutige zersplitterte Weltgesellschaft eine philosophische Gerechtigkeitstheorie die Verstockheit kultureller Unterschiede nicht einfach akzeptieren darf. ${ }^{21}$ Stattdessen schlägt er vor, universelle Menschenrechte vom Standpunkt eines fallibilistischen Bewusstseins zu verteidigen, das zugesteht, „dass das im Okzident entwickelte Verständnis der Menschenrechte prima facie gut begründet ist und - auch wenn es im Lichte überzeugender Einwände korrigiert und gegebenenfalls erweitert werden muss - universelle Zustimmung verdient“"22.

17 Die Bedeutung von Glaubensfragen für Rawls’ Werk ist seit der Veröffentlichung seiner Doktorarbeit klarer geworden; vgl. Rawls (2009). Vgl. auch Benhabib (2019), meine Rezension von Andrius Gališankas Buch John Rawls: The Path to A Theory of Justice (Gališanka 2019).

18 Finlayson (2019); Finlayson/Freyenhagen (2011).

19 Vgl. Rawls (1995). In diesem Essay replizierte Rawls auf die Diskussion seiner Arbeiten in Habermas (1992), die ihm in verschiedenen Übersetzungen zugänglich gemacht worden waren. Vgl. auch Baynes (1991), McCarthy (1994) u. Forst (1996).

20 Habermas (2019a), 99.

21 Ebd.

22 Ebd. (Hervorh. im Orig.). Habermas stellt Karl Jaspers' Offenheit für den Dialog mit Theologen Rawls' Enthaltung von einer solchen Auseinandersetzung gegenüber und lobt Jaspers' Kon- 
Es ist unmöglich, den Feinheiten von Habermas' Erzählung gerecht zu werden; ihr Blickwinkel und ihre Gelehrtheit sprengen die Grenzen jedes Einzelkommentars. Dennoch bleibt eine hartnäckige Frage: Wie könnte ein fallibilistisches Bewusstsein allgemeiner Menschenrechte, offen für das Lernen von anderen kulturellen Traditionen in einer Weltgesellschaft, allein in den Begriffen einer Genealogie der abendländischen Vernunft errichtet werden? Warum kann eine Erzählung der Begegnung von Glauben und Wissen in der westlichen Tradition eine Antwort auf die heutzutage die Menschheit herausfordernden Rätsel bieten, darunter der Zusammenstoß von globalem Kapitalismus, Familienleben und religiösen Gemeinschaften; die Verbreitung von Robotik, der Verlust von Arbeitsplätzen und sinnvoller Arbeit; die Aneignung von Gentechnologie durch die Superreichen (z. B. Elon Musk), die eines Tages sehr wohl die Fähigkeit haben könnten, ihren Nachwuchs anzufertigen? Wie können so allgemeine Fragestellungen durch eine geographisch und kulturell so eingegrenzte Erzählung beantwortet werden?

Ein Schlüssel dazu, die Antwort freizulegen, ist es, Habermas' Buch mit Hegels Phänomenologie des Geistes (1807) zu vergleichen. ${ }^{23}$ Auch Hegel stellte die Errungenschaften der Moderne und den Ort seiner eigenen Philosophie in ihrem Zeitalter als eine große Erzählung, rekonstruiert als Lernprozess, dar. ${ }^{24}$ Ich möchte jedoch argumentieren, dass sowohl Hegels phänomenologische als auch Habermas' rekonstruktive Methode die Fehlschlüsse einer Art von Historizismus wiederholen. ${ }^{25}$ Sie behandeln Entwicklungsprozesse, als würden sie durch ein hypostasiertes Kollektiv in einer homogenen Zeit vollzogen, die als ein „Gefäß“ der Entwicklung betrachtet wird, statt Zeitlichkeit als lebendige, diskontinuierliche und widersprüchliche Erfahrung zu verstehen. ${ }^{26}$ Ein solcher Historizismus hat zur Folge, dass die Stimmen jener anderen, die beim „Lernprozess“ außen vor

zept des gemeinsamen Ursprungs aller „starken“ Traditionen, da diese aus der Revolution der Weltbilder in der Achsenzeit hervorgehen (ebd., 101).

23 Vgl. auch Gordon (2021).

24 Vgl. Habermas (2019b), 473: „Hegel ist nicht nur der erste geschichtlich denkende Philosoph; er nimmt auch als Erster ein philosophisches Interesse an der Entstehung der europäischen Moderne - und behandelt das Kantische Thema vernünftiger Freiheit als den Kern dieser bahnbrechend neuen theoretischen Fragestellung “ (Hervorh. im Orig.).

25 Habermas diskutiert den deutschen Historismus im Lichte der Betonung von Individualität und der Singularität historischer Geschehnisse (vgl. ebd.). Ich verwende den Ausdruck in dem von Walter Benjamin begründeten Sinne, der die geschichtlichen Zeiten betrachtet, als wären sie „Perlen auf einem Rosenkranz“ und die verschiedenen Zeitlichkeiten verschiedener Begegnungen mit der Geschichte übersieht.

26 Für eine Kritik solcher Begriffe von Zeitlichkeit vgl. Benjamin (1992). 
bleiben, während er unaufhaltsam voranschreitet, ignoriert und ausgeklammert werden. Ganze Landstriche menschlicher Gesellschaften werden in das „Wartezimmer" der Geschichte verbannt und es gerät in Vergessenheit, dass wir dieselben und dennoch unterschiedliche geschichtliche Zeitlichkeiten bewohnen und dass die Geschichte der globalen Moderne nicht länger nur über die Begegnung von Vernunft und Glauben im Okzident allein erzählt werden kann.

Ich möchte betonen, dass diese Analogie keine ist, die Hegels Metaphysik oder Ontologie verteidigt; offensichtlich kann nichts weiter von Habermas' fallibilistischer Konzeption kommunikativer Vernunft entfernt sein als Hegels Anspruch auf „absolutes Wissen“. Ich stelle lediglich die Form von Zeitlichkeit in Frage, die durch alle Lernprozesstheorien vorausgesetzt werden muss. In dieser Hinsicht hatte Hegel ein komplexes Argument zu bieten, das unsere Aufmerksamkeit verdient hat.

\section{Hegels Phänomenologie des Geistes als Vorläufer von Habermas' Auch eine Geschichte der Philosophie}

Hegel wird in Band 2 von AGP umfassend diskutiert, ${ }^{27}$ der dem Nachziehen der Spuren der „[v]ernünftige[n] Freiheit“ gewidmet ist, so wie sie aus dem Dialog zwischen Glauben und Wissen hervorgeht. Habermas konzentriert sich auf eine grundlegende Spannung in Hegels Philosophie: Auf der einen Seite verdankt sich die dialektische Ausfaltung des BEGRIFFs ${ }^{28}$ der alten Lehre des „Nezessitarianismus“, das heißt, der Einsicht, dass das „Wirkliche vernünftig ist“. „Andererseits feiert der eurozentrische Geschichtsphilosoph die Unabhängigkeit des selbstbewussten, sich selbst bestimmenden und verwirklichenden Subjekts“. ${ }^{29}$ Für Habermas sind Hegels soziohistorische Einsichten in die Spannungen und Strukturen der modernen Gesellschaft unentwirrbar verflochten mit dieser metaphysischen Hypothek, die auch danach strebt, die Wahrheiten der Religion in dialektischer Philosophie aufzulösen. Habermas hat Einwände gegen gegenwärtige Hegelinterpretationen, wie sie Terry Pinkard und Robert Pippin vorschlagen ${ }^{30}$, die sich

27 Habermas (2019b), 468-555.

28 Wörter in Kapitälchen sind im englischen Original groß geschrieben (Anm. d. Übers.).

29 Ebd., 509.

30 Pinkard (1996); Pippin (2008). 
des Absoluten Geistes durch eine „neo-pragmatische Enthauptung“ entledigen. ${ }^{31}$ Ich möchte in diese Debatte über gegenwärtige Hegelinterpretationen nicht einsteigen. Meine Absicht ist eine viel eingegrenztere. Wie Habermas zugesteht, war es Hegel, der als erster vorschlug, dass wir das Mittel eines Lernprozesses verwenden könnten, um die Errungenschaften der Moderne zu verstehen. Wie in Hegels Phänomenologie muss auch für Habermas’ Methode der „vernünftigen Rekonstruktion“ das Zusammenspiel zwischen dem Standpunkt des Beteiligten und dem des Beobachters so verstanden werden, dass es sich im Lichte guter Gründe entfaltet und nicht einfach so beschrieben wird, als wäre es eine plausible Geschichte wie jede andere. ${ }^{32}$ Dieses Argument möchte ich hier beleuchten.

Hegels Phänomenologie beginnt als erkenntnistheoretisches Argument bei seinen Vorgängern, besonders Kant, und setzt sich als Bildungsroman eines Protagonisten namens „das Bewußtsein“33 fort. Für Hegel ist Bewusstsein anfangs zunächst als Repräsentation des Überzeugungssystems eines Durchschnittsmenschen zu beschreiben, der einer Korrespondenztheorie der Wahrheit anhängt. Das Bewusstsein glaubt ganz einfach, dass Wahrheit von etwas handelt, was ihm äußerlich ist, und denkt, dass Wissen entweder ein Instrument oder ein Medium ist, wodurch es diese Wahrheit erreichen kann. Das Bewusstsein gerät erst ins Schwimmen, sobald es die Gewissheit jener Wahrheit einem anderen nachweisen oder mitteilen soll. Woher kommt diese Notwendigkeit, Gewissheit nachzuweisen? ${ }^{34}$ Zinkt Hegel nicht von Anfang an die Karten, indem er verlangt, dass Wahrheit mitgeteilt und mitteilbar sein müsse? Zweifellos, doch wir, die wir dem Fortschreiten des Abenteuers des Bewusstseins zuschauen, sind nicht bloß Beobachter, da wir in die Geschichte des Bewusstseins hineingezogen werden, insoweit wir auch einige Annahmen des Bewusstseins teilen. Hegel etabliert die Inadäquatheit bestimmter Wahrheitsbegriffe über ein dialogisches Zusammenspiel zwischen dem Standpunkt des „Wir“ und des Bewusstseins. Was auch immer der alte Hegel über das Absolute Wissen gesagt haben mag: Die Absicht der Phänomenologie ist es, die Wahrheitssuche als einen dialogischen Lernprozess, der sich zwischen dem Leser und dem Standpunkt des Bewusstseins entfaltet, zu zeigen.

31 Habermas (2019b), 509.

32 Ders. (2019a), 139.

33 Hegel (1986).

34 Hegels epistemisches Argument ist, dass eine Wahrheitsbehauptung nicht bloß von einem subjektiven Geisteszustand handelt, sondern mit anderen geteilt und kommuniziert werden muss. Sellars (1997), Rorty (2009) und Brandom (1994) haben diese hegelsche Einsicht zu einer weitreichenden Kritik von Empirismus, Mythos des Gegebenen und Zuschauertheorie des Wissens ausgearbeitet. Dewey (2008) hatte diesen Weg bereits eingeschlagen. 
Die Methode der Phänomenologie wird noch erhellender für das Verständnis von Habermas' Zielen in AGP, wenn wir das Folgende bedenken: Die Überzeugungen des Bewusstseins über Wahrheit und Gewissheit bilden zusammen „eine Gestalt des Bewußtseins“ - die Erfahrung, eine der Wahrheit angemessene Gewissheit zu artikulieren zu versuchen, treibt das Bewusstsein einem neuen Wahrheitsobjekt entgegen. Wenn das Bewusstsein versucht, die Gewissheit über seine Wahrheitsbehauptung mitzuteilen, „leidet [es] also diese Gewalt, sich die beschränkte Befriedigung zu verderben, von ihm selbst“. ${ }^{35}$ Der Prozess, zu sagen oder zu zeigen, was Wahrheit ist, beweist selbst, dass Wahrheit nicht das ist, was das Bewusstsein dachte, dass es sei. ${ }^{36}$

Die Phänomenologie ist eine Erzählung davon, wie die Gestalten des Bewusstseins zunehmend komplexe epistemologische, gesellschaftliche und kulturelle Lebensformen werden. Mit dem Wandel der Erfahrungen des Bewusstseins wandeln sich auch sein Objekt und seine Subjektivität: Von den epistemischen Begegnungen der ersten drei Kapitel (namens „Die sinnliche Gewißheit“, „Die Wahrnehmung“ und „Kraft und Verstand, Erscheinung und übernatürliche Welt“) aus geht das Buch zu Selbstbewusstsein und Begehren über, zu Arbeit und der Herr-Knecht-Dialektik, dann zu Vernunft, Geist, Kunst, Religion und schließlich zu Absolutem Wissen, das sich als Rekapitulation des Ganzen herausstellt.

Was aus Hegels Darstellung einen Lernprozess macht und nicht einfach nur eine brillant erzählte Geschichte, ist, dass wir, der Erzähler und die Leserin, die dem Bewusstsein zuschauen, herausfinden, dass der Übergang von einer Form von Bewusstsein zur anderen durch „Erfahrung“ geschieht, also als Lernprozess. Doch nur wir wissen das: Das Bewusstsein weiß nicht, wie oder warum es den Gegenstand seines Wissens oder seine Gewissheit geändert hat. All dies findet „hinter seinem Rücken“ statt. ${ }^{37}$ Für Hegel ist der Übergang von einer Form von Bewusstsein zu einer anderen genau deswegen ein Lernprozess, weil, wie Habermas fordert: „Während Anpassungsprozesse aus der Beobachterperspektive unmittelbar zugänglich sind, muss der beobachtende Soziologe Lernprozesse aus der Beteiligtenperspektive erst nachvollziehen, bevor er sie beschreiben kann. “38.

Um von einem Lernprozess sprechen zu können, und nicht nur von einem evolutionären Anpassungsprozess, bei dem menschliche Gemeinschaften ihre Praktiken und Weltsichten in Reaktion auf funktionale Imperative in der Umwelt verändern, muss es eine Art von Subjekt geben, ein Wir, das fähig ist sowohl

35 Hegel (1986), 74.

36 Taylor (1972).

37 Hegel (1986), 80.

38 Habermas (2019a), 139, Hervorh. S. B. 
zu lernen als auch zu vergessen. Obwohl Hegel für sein Konzept von Geist, der sich selbst in der Geschichte entfaltet, viel Hohn und Spott empfangen hat, braucht Geist nicht als ein Großsubjekt begriffen $\mathrm{zu}$ werden, sondern, wie der junge Habermas wohl wusste, kann Geist auch als eine intersubjektiv integrierte Gemeinschaft von Lernenden gesehen werden. ${ }^{39}$ Im Vorwort zur Phänomenologie sagt Hegel explizit, dass die Leser seines Buchs die modernen Individuen im Kielwasser der Französischen Revolution sind, die des Heraufziehens der Neuen Zeit - oder: der Moderne - gewahr sind.

Wenn diese Analogie zur Phänomenologie erhellend ist, dann ist die Frage: Wer ist das Wir, das in Habermas' AGP das Lernen und Vergessen übernimmt? Habermas' glänzende Nacherzählung der Philosophiegeschichte als Zusammenspiel zwischen Glauben und Wissen ist am Ende des Tages eine sehr selektive Geschichte der intellektuellen Wandlungen der okzidentalen Menschheit. Die protestantische Revolution nimmt in dieser Erzählung einen besonderen Ort ein. Luther gibt der Trennung zwischen der Abrechnung eines Individuums mit seinem eigenen Glauben in der Anwesenheit Gottes und der institutionellen Staffage einer zunehmend korrupten katholischen Kirche Ausdruck. Das Drama zwischen Katholizismus und Protestantismus ist ein zentraler Wendepunkt im zweiten Band des Buches. Die Ideale der kantianischen sittlichen Autonomie gehen aus dieser Begegnung hervor. Das Judentum verschwindet in der Geschichte und wird in einer ,egalitär-universalistische[n]“ und „egalitär-individualistischen“ - und, so möchte ich sagen, hochgradig protestantischen - Form von Moralität aufgehoben. ${ }^{40}$

In den letzten Jahren sind Vorwürfe des „Eurozentrismus“ gegen die Tradition der Kritischen Theorie im Allgemeinen und AGP im Besonderen erhoben worden. ${ }^{41}$ Habermas ist sich dieser Einwände bewusst und räumt die von westlichen, sich modernisierenden Nationen über den Rest der Welt entfesselte imperialistische Herrschaft mehrfach ein. ${ }^{42}$ Doch meine Frage ist eine methodologische, die bis auf die Habermas-Luhmann-Debatte zurückgeht: Wenn die Theorie des kommunikativen Handelns eine Kritik einer systemtheoretischen Beschreibung ist und sich weigert, die Gesellschaft als funktional integrierte Subsysteme zu betrachten, die lediglich auf ihre Umwelt reagieren, um ihre funktionalen Fähigkeiten zu verbessern, dann können die Träger des Lernprozesses in der Theorie

39 Vgl. ders. (1968). Der Titel der dritten Zwischenbetrachtung in AGP 2 ist „Vom objektiven Geist zur kommunikativen Vergesellschaftung erkennender und handelnder Subjekte“ (ders. 2019b, 559).

40 Ebd., 82.

41 Allen (2016); Bloch (2020).

42 Habermas (2019a), 110. 
des kommunikativen Handelns nur ein Wir sein. Doch wer sind wir? Habermas scheint zwischen einem neo-hegelianischen und einem neo-evolutionären Lernensbegriff zu schwanken. Das heißt, dass der Lernprozess entweder einer ist, der als eine Folge „guter Gründe“ stattfindet, die aus einer Erfahrung resultieren, die Individuen machen, oder der Lernprozess ist eigentlich eine falsche Bezeichnung für Anpassungen, die gesellschaftliche Systeme anonym durchlaufen, um fähig zu sein, sich bestimmten Umgebungen anzupassen und darin zu überleben.

Unsere Frage kommt daher darauf zurück: Was gibt diesem Lernprozess eine allgemeine Bedeutung, die im Großen Beiträge zu Fragen der Weltgesellschaft in einem postmetaphysischen Zeitalter zu liefern vermag? Oder: Was verwandelt das Faktum der Modernität, das sich „mit vorgehaltener Waffe oder per Kanonenboot oder Kampfhubschrauber“43 über den Rest der Welt ausbreitete, in eine normative Erzählung für ein „Wir“, dessen Identität und Stimmen nach wie vor unbestimmt sind? Die Normativität der Moderne verlangt die Einbindung vieler anderer Stimmen, die mit der „Drohung der Exklusion, Marginalisierung, Subordination“44 kämpfen.

Es ist kein Zufall, dass Habermas' Titel auf Herders Auch eine Philosophie der Geschichte zur Bildung der Menschheit ${ }^{45}$ anspielt. Und Habermas' Philosophiegeschichte wird, malgré elle, zu einer Geschichtsphilosophie, solange der Lernensbegriff nicht weiter geklärt ist. Kant kannte die Schwierigkeiten universeller Geschichtsschreibung sehr genau, nicht nur, weil er wusste, wie viel er und seine Zeitgenossen über die Menschheit noch nicht kannten und wussten. Deswegen behauptete er, dass er von der Menschheit nur als einem „spekulativen“ Subjekt einer mit weltbürgerlicher Absicht geschriebenen Geschichte sprechen konnte. ${ }^{46}$ Das Subjekt einer solchen Geschichte war eine kommende Menschheit - „une humanité à venir“.

Der Vergleich mit Hegels Phänomenologie des Geistes ist in mehr als einer Hinsicht nützlich. Da sich der Standpunkt des Bewusstseins schließlich an den des „Wirs“ annähert und die Erzählung im Text als eine selektive historische Rekonstruktion der Grenzen der europäischen Aufklärung und ihrer Überwindung (Aufhebung) erkennbar wird, bricht die Asymmetrie zwischen dem, was das Bewusstsein als Wahrheit behauptet, und dem, von dem der Beobachter weiß, dass es die Wahrheit ist, zusammen. Die Gestalt, oder der Rahmen, des Bewusstseins

\footnotetext{
43 McCarthy (2015).

44 Ebd.

45 Herder (2020).

46 Kant (2019). Die Idee einer „spekulativen Geschichte“ wurde erstmals von Rousseau (2019) entwickelt, als dieser eine hypothetische frühe Anthropologie der Menschheitsentwicklung vorschlug.
} 
wird zunehmend eine, die auch wir bewohnen. Wir werden Widerparte in einer Unterhaltung, oder, wie Habermas einmal gesagt hat, „in einem Aufklärungsprozeß gibt es nur Beteiligte“ ${ }^{* 47}$. Wenn diese Asymmetrie zusammenbricht und es nur noch symmetrisch angeordnete Partner gibt, von denen keiner einen privilegierten Standpunkt einnimmt, welche philosophische Tragweite hat der Begriff eines Lernprozesses dann noch? Warum genau soll die Genealogie postmetaphysischer Vernunft ein überzeugendes Argument für die Geltung eines solchen Standpunktes sein? Jede Art von Genealogie geht aus einer Asymmetrie des Wissens zwischen jenem, der sie betreibt, und dem analysierten Objekt hervor. Unter der Bedingung symmetrisch angeordneter Partner haben wir den äußeren Standpunkt von jemandem, der urteilt, was als Lernen gelten kann, nicht mehr. Ist Genealogie noch plausibel, wenn diese Asymmetrie einmal zusammengebrochen ist?

Diese Herausforderung kommt für Habermas in seiner Auseinandersetzung mit David Hume auf. Er gesteht zu, dass Hume derjenige Denker wäre, der behaupten könnte, dass all die zur Rekonstruktion der Genealogie der postmetaphysischen Vernunft unternommenen Schritte eine „verständliche Illusion“48 seien. Hume repräsentiert einen humanistischen Naturalismus, nach wie vor von der griechischen und römischen Tradition beeinflusst, und distanziert sich von christlich geprägten heilsgeschichtlichen Fortschrittserzählungen. Sein Skeptizismus ist daher immun gegenüber Illusionen eines vernünftigen Lernprozesses. Selbst wenn dies so ist, können wir heutzutage dennoch mit den Erben Humes nur in einem geteilten „Raum der Gründe“49 diskutieren, und die Tatsache, dass der eigene Standpunkt das Ergebnis eines rational rekonstruierten Lernprozesses ist, wird seiner Geltung wenig hinzufügen.

Lassen Sie mich meine bisherige Argumentation zusammenfassen: Ich finde die Analogie zu Hegels Phänomenologie erhellend, da die Methode, die Errungenschaften der Moderne als Folge eines Lernprozesses darzustellen, Hegels wichtigster Beitrag ist. Sobald wir uns chronologisch der Moderne nähern, beginnt Hegels Erzählung in den Kapiteln 6 und 7 der Phänomenologie der Laufbahn des GEISTES in der europäischen Aufklärung, der Französischen Revolution und danach in seiner Verkörperung in Kunst und Religion $\mathrm{zu}$ folgen. All dies stellt eine Näherung an eine wiedererkennbare Chronologie europäischer Geschichte dar, und Hegels Erzählung bleibt keine bloße hypothetische Geschichte. Dennoch müssen wir genau hier Hegel ebenso wie Habermas fragen: Wer ist das Subjekt-

47 Habermas (1978), 45.

48 Ders. (2019b), 210.

49 Brandom (1994). Vgl. z. B. Annette Baiers hochgradig einflussreiche Arbeit zu Hume in Baier (1987) und dies. (1993). 
Objekt dieser Geschichte? Zudem reichen, da die Asymmetrie zwischen demjenigen, dessen Erfahrung erzählt wird, und dem Erzähler, dessen Geschichte erzählt wird, zusammenbricht, weder Genealogie noch Phänomenologie aus, um die Normativität der Moderne zu etablieren.

Ich möchte vorschlagen, dass Erzählungen dieser Normativität der Moderne der Pluralität von Stimmen, die üblicherweise nicht gehört werden, gerecht werden müssen. Die „Einbeziehung des Anderen“ bedeutet die Anerkennung der Stimme des Anderen im Dialog der Moderne. In den letzten Jahrzehnten hat feministische Forschung viele Werke hervorgebracht, die die Geschichte der westlichen Philosophie erzählen, indem sie die Stimmen von Frauen in diese Erzählung einbinden. ${ }^{50}$ Und wenn und falls wir dies tun, geht daraus eine andere Anordnung des Verhältnisses von Vernunft und Gefühl, Rationalität und Verkörperung, Allgemeinem und Besonderem hervor - neben vielen anderen Themen. Diese Beiträge führen uns, wie ich in zahlreichen Publikationen argumentiert habe, dazu, eine neue Anordnung des ,verallgemeinerte[n]“ und „konkrete[n] Andere[n]“51 zu bedenken. Die Einführung der Stimmen von Frauen in die Erzählung der Moderne hat nicht nur Konsequenzen für Erkenntnistheorie und Moraltheorie, sondern auch dafür, wie wir über das Thema Menschenrechte nachdenken. Welche verborgenen Annahmen über den menschlichen Körper, seine Lebenszyklen, seine Sexualität haben das herrschende Verständnis der Rechte innehabenden Person gelenkt? Wie ändern sich unsere Begriffe von menschlicher Gleichheit, wenn die Person nicht nur ein erwachsener, wehrfähiger Mann ist, sondern eine junge, schwangere, minderjährige Mutter? In der Tat muss man es, sofern, wie Habermas unterstellt, eine Verteidigung der Menschenrechte vom Standpunkt eines falliblen Bewusstseins zugesteht, „dass das im Okzident entwickelte Verständnis der Menschenrechte prima facie gut begründet ist ${ }^{\text {“52 }}$, bedauern, dass die Stimmen von Frauen nicht in Habermas’ Erzählung eingegangen sind.

Nicht nur die Stimmen von Frauen werden in dieser erzählenden Darstellung der Moderne ausgelöscht. Der transkulturelle Dialog, der in einer Weltgesellschaft eigentlich stattfinden sollte, wird ebenso vergessen.

50 Lloyd (2004); Pateman (1988); Okin (1979 u. 1991); Elshtain (1981); Nussbaum (1999); Benhabib/Nicholson (1987). Zu Forschung innerhalb der Tradition der Kritischen Theorie, eng oder breit konzipiert als die Psychoanalyse, foucaultsche Theorie sowie derridasche Dekonstruktion einschließend, vgl. Benhabib/Cornell (1987); Benhabib et al. (1993 u. 1996); Cornell (1999); Fraser (1989 u. 1997); Fraser/Honneth (2003); Fraser/Jaeggi (2018).

51 Benhabib (1995).

52 Habermas (2019a), 99; Hervorh. im Orig. 


\section{3 „Europa als Provinz“}

In den letzten paar Jahrzehnten ist eine Art historischer Forschung aufgekommen, deren Ziel es ist, die Erzählung einer globalen Moderne als mehrstimmig, konflikthaft und uneinig zu präsentieren. 2000 veröffentlichte Dipesh Chakrabarty Provincializing Europe. Dieses Buch ist eines der eloquentesten innerhalb eines umfangreichen geschichtswissenschaftlichen Korpus, der eine solche komplexe und vielschichtige Darstellung der europäischen Moderne auf ihrem Weg zu globalen Ausmaßen hervorbringt. ${ }^{53}$ Ebenso wie die oben genannten Beiträge feministischer Gelehrter verpflichtet uns dieses Korpus dazu, die Idee eines sich einheitlich entfaltenden Lernprozesses in Frage zu stellen. Für einige postkoloniale Theoretikerinnen mag die Geschichte der Moderne von ihren Rändern her zu schreiben, indem sie die Stimme des „Subalternen“ einbeziehen, zu einer Zurückweisung der Moderne insgesamt führen. Chakrabartys Buch ist philosophisch genau deswegen wichtig, weil „Europa als Provinz“ für ihn nicht heißt, das Erbe und die Leistungen der europäischen Moderne zu verwerfen. Ganz im Gegenteil. Chakrabarty verortet sich innerhalb einer hermeneutisch-genealogischen Tradition und versucht, HansGeorg Gadamers Hermeneutik mit Michel Foucaults genealogischer Methode zu versöhnen. ${ }^{54}$ Seine Kritik des Historizismus und seine Rekonstruktion der Mehrstimmigkeit des Dialogs der Moderne lohnen ein ausführliches Zitat:

Das Phänomen der „politischen Modernität“ - das heißt der Herrschaft von moderner staat-
licher Institutionen, Bürokratie und kapitalistischen Unternehmen - ist überall auf der Welt
unmöglich zu denken, ohne bestimmte Kategorien und Begriffe aufzurufen, deren Genealo-
gien tief in die intellektuellen und sogar theologischen Traditionen Europas zurückreichen.
Begriffe wie Bürgerschaft, der Staat, Zivilgesellschaft, Öffentlichkeit, Menschenrechte,
Gleichheit vor dem Gesetz, das Individuum... und so weiter, sie alle tragen die Last europäi-
schen Denkens und europäischer Geschichte. Man kann einfach nicht über politische Moder-

53 Chakrabarty unterstreicht, man könne nicht „das Lokale entleeren, indem man es irgendeiner abstrakten Allgemeinheit assimiliert“ (Chakrabarty 2008, 18). Stattdessen sei es das Ziel, „affektive Geschichten“ (ebd.) zu schaffen. Warum genau Chakrabarty Heideggers Philosophie für einer solchen Analyse zuträglich hält, ist mir ein Rätsel, denn wenn es einen Denker gibt, dessen furchterregende Abstraktionen die Lebenswelt bis zur Unkenntlichkeit reduzieren, dann ist es Heidegger mit seinen philosophischen Neologismen.

54 Chakrabarty achtet nicht immer darauf, die Unterschiede zwischen divergierenden Denkern herauszuarbeiten. Foucaultsche Genealogie ist, in Paul Ricœurs berühmten Worten, eine Anwendung der Hermeneutik der „Schule des Zweifels“ (Ricœur 1993, 41), während gadamersche Hermeneutik auf eine Unterhaltung abzielt, die sich über Jahrhunderte hinweg durch eine „Wirkungsgeschichte“ entfaltet, in der wir symmetrisch angeordnet sind. 
nität nachdenken ohne diese und andere verwandte Begriffe, die ihre höchste Ausprägung im Laufe der Europäischen Aufklärung und des neunzehnten Jahrhunderts gefunden haben. ${ }^{55}$

Diese Begriffe sind in die Lebenswelt unserer globalen politischen Moderne eingebettet. Doch wir müssen wachsam gegenüber der Politik des Historizismus werden. „Historizismus“ jener Art, wie Hegel und Habermas sie mittels des Begriffs eines Lernprozesses vertreten, „postulierte historische Zeiten als ein Maß der kulturellen Distanz (wenigstens in der institutionellen Entwicklung), von der unterstellt wurde, dass sie zwischen dem Westen und dem Nicht-Westen bestünde“ ${ }^{\text {"56 }}$. Dies führte zu einem Begriff globaler geschichtlicher Zeit als „zuerst in Europa, dann anderswo“57. Historizismus ist ,jemandes Art, ,noch nicht jetzt zu jemand anderem zu sagen“58.

Eine große Stärke von Chakrabartys Analyse ist es, dass sie aufzeigt, wie Eliten auch in nichtwestlichen Ländern diesen Wartezimmerblick auf die Geschichte verwendet haben, um ihren eigenen Völkern zu sagen: „nicht jetzt: Ihr seid noch nicht bereit für das allgemeine Wahlrecht, für Frauenrechte, für Demokratie“. Der Dialog der globalen Moderne findet demnach nicht zwischen den holistischen Kategorien von Okzident und Orient statt, sondern zwischen jenen, die, ganz gleich wo, menschlicher Gleichheit und Würde verpflichtet sind, und anderen, die die historistische Logik nutzen, um die Volksmassen dazu zu verdammen, im Vorzimmer der Geschichte zu warten. ${ }^{59}$

Solche historisch-philosophischen Beschreibungen zwingen uns dazu, die Idee eines einsträngigen Lernprozesses von zwingender Gerichtetheit in Zweifel zu ziehen. Wer lernt und wer vergisst? Wir müssen eher fragen: Wer lernt anders? ${ }^{60}$

55 Chakrabarty (2008), 4.

56 Ebd., 7.

57 Ebd.

58 Ebd., 8.

59 In Chakrabartys eigener Arbeit als marxistischer Historiker Indiens führte das Verwerfen dieses Historismus zu seiner Entdeckung der politischen Handlungskraft der Bauernschaft. Die Modernisierungs-Ansicht besagt, dass der Bauer zum Bürger erzogen werden muss: „[T]he other is the peasant who, despite his or her lack of formal education, is already a citizen“ (ebd., 10). Die Entdeckung der politischen Handlungskraft der Bauernschaft befeuerte eine der größten Revolutionen des 20. Jahrhunderts, nicht die russische, sondern die chinesische Revolution. Die Bauern, bis dahin für „rückständig“ oder sogar, in Marx’ berühmtem Wort, für einen „Kartoffelsack“ gehalten (Marx 1960, 198), wurden zu Akteuren in einer modernen politischen Geschichte, was viele antikoloniale Befreiungsbewegungen inspirieren sollte. Es ist unmöglich, heute die Geschichte der allgemeinen Menschenrechte zu schreiben, ohne auch das Erbe dieser Bewegungen zu betrachten; vgl. Anghie (2005).

60 Yirmiyahu Yovels The Other Within: The Marrano: Split Identity and Emerging Modernity (Yovel 2009) ist ein brillantes Werk in diesem Genre. Yovel bespricht, inwiefern das Schicksal 
Für manche mag die Moderne eine neue Wiederverzauberung und nicht bloß eine Rückkehr zur alten Kirche, wie Weber dachte, eingeläutet haben. Doch dies bedeutet nicht, dass wir die kognitiven Errungenschaften der modernen Wissenschaft verwerfen sollten - Falsifizierbarkeit, Reflexivität und epistemische Bestätigung durch eine Forschungsgemeinschaft (community of inquiry). Es bedeutet, dass wir die Stimmen jener, die diese epistemischen Werte mit den Göttern ihrer alltäglichen Lebenswelten zu versöhnen versuchen, ernster nehmen müssen. ${ }^{61}$ Geschichte ist nicht das „Wartezimmer“ dieser anderen, die darum bitten, hineingelassen zu werden. Wir bewohnen dieselben und dennoch andere geschichtliche Zeitlichkeiten, und die Geschichte der globalen Moderne kann nicht länger alleine als die Auseinandersetzung von Vernunft und Glauben im Okzident erzählt werden.

Ironischerweise hatte Hegel hierfür ein gewisses Gespür. Susan Buck Morss' meisterhafte Rekonstruktion der berühmten „Herrschaft und Knechtschaft“62 enthüllt, dass Hegel den Ausbruch der Revolution in Haiti (1791-1804) unter der Führung von Toussaint l'Ouverture eifrig und enthusiastisch verfolgte. Durch Zusammenstöße zwischen den Sklaven, den Kolonisatoren und der britischen und französischen Armee errangen haitianische Revolutionäre ihre Unabhängigkeit und schufen das erste von ehemaligen Sklaven begründete Land. ${ }^{63}$ Hegel, von dem berichtet wird, dass er sein Leben lang einen Toast auf die Befreiung der Bastille am 14. Juli 1789 ausgebracht habe ${ }^{64}$, arbeitete gerade den Kampf der Sklaven in seine glänzende Darstellung in der Phänomenologie ein, als Napoleon in Jena einzog.

der Marranos - Juden, die in der Zeit der Inquisition gezwungen wurden, zum Katholizismus zu konvertieren, aber im Geheimen Aspekte ihrer jüdischen Identität zurückbehielten - emblematisch für die Fluidität und Heterogeneität von Identitäten in der Moderne ist. Statt den Juden als dem „traditionellen Anderen“ ist der Marrano der „Andere im Inneren“, den Machthaber aus dem politischen Körper zu entfernen suchen, jedoch ohne großen Erfolg. Stattdessen haben die meisten Erzählungen der Moderne, Habermas' eingeschlossen, die Tendenz, das Reformjudentum dem Protestantismus einzuverleiben.

61 Angesichts der Verschärfung der ökologischen Krise und der klareren Erkenntnis des irreversiblen Schadens, der dem Planeten im Anthropozän zugefügt wurde, müssen wir mehr Respekt haben vor (und lernen von) den Weisen, mit und in der Natur zu leben, wie sie von indigenen Völkern praktiziert werden, für die die Natur in mancherlei Hinsicht verzaubert bleibt. Von der Forst- über die Landwirtschaft bis hin zur Medizin ist die Reduktion von lebendem Raum und lebendem Körper zu gebrauchbarer toter Materie für einen Großteil des Leidens, das wir heutzutage auf einer planetarischen Ebene erfahren, verantwortlich.

62 Buck-Morss (2009).

63 Für eine Diskussion des Erbes der haitianischen Schulden und die erdrückenden Verpflichtungen, unter denen Haiti bis heute leidet, vgl. z. B. Daut (2020).

64 Vgl. Pinkard (2000), 451. 


\section{Schluss}

Ich möchte zwei habermasianische Einwände gegen mich selbst erheben: Zunächst lässt sich argumentieren: Während es sicherlich eine wünschenswerte Aufgabe ist, die Erzählung der globalen Moderne komplexer zu gestalten, sodass die Stimmen der Anderen als Dialogpartner eingebracht werden, eine Aufgabe, die künftige Gelehrtengenerationen werden angehen müssen - warum ist sie philosophisch bedeutend? Läuft man mit ihr nicht Gefahr, Philosophie in eine Form von historisch-kulturwissenschaftlichen Studien zu transformieren?

Die Grenzen des Wissens zu patrouillieren ist eine vergebliche Aufgabe, und insbesondere die Kritische Theorie kann sich damit nicht aufhalten. Wenn die Logik des Gegenstandes, „die Sache selbst“ es erfordert, dann verlangt die Forschung, dass wir uns damit beschäftigen. Kritische Theorie hat stets einen Dialog zwischen Philosophie und Gesellschaftswissenschaften praktiziert, und wir stehen alle in der Schuld von Habermas' bahnbrechenden Werken zur Genealogie der Moderne, angefangen mit Strukturwandel der Öffentlichkeit, die solche Grenzüberquerungen zeitigten. Genauso, wie Habermas das Bürgertum dafür kritisierte, die Stimmen von Arbeitern und Frauen aus der aufkommenden bürgerlichen Öffentlichkeit auszuschließen, müssen wir uns heute bemühen, all diese Stimmen plus Stimmen aus dem Globalen Süden in unsere Narrationen der Moderne einzuschließen.

Zweitens: Wie steht es mit der Frage der Geltung? Vielleicht muss die Genealogie der „vernünftigen Freiheit“ etwas anders erzählt werden, indem man die Stimmen der Ausgeschlossenen einbezieht, aber schmälert dies die Geltung des Projekts vernünftiger und kommunikativer Freiheit? Und in ähnlicher Weise schmälert die Tatsache, dass Hegel ein historistisches Geschichtsverständnis hat, gleichermaßen die Geltung seiner erkenntnistheoretischen Argumente gegen die Korrespondenztheorie der Wahrheit? Die Antwort auf beide Fragen ist „nein“: ${ }^{65}$

65 Dies unterscheidet meine Kritik von der Amy Allens (2016): Ich denke, dass wir sowohl die normativen als auch die kognitiven Errungenschaften der Moderne verteidigen und nicht darum verlegen sein sollten, dass die Ausbreitung der allgemeinen Menschenrechte, unbenommen ihres Gebrauchs und Missbrauchs durch viele für ihre eigenen imperialistischen und neokolonialen Ziele, einen Fortschritt für die Conditio humana darstellt. Und Kriterien wie Reflexivität, Falsifizierbarkeit und Revidierbarkeit durch eine Community of Inquiry sind ebenfalls Anzeichen von kognitivem Fortschritt. Das Thema des geschichtlichen Fortschritts als solchem ist hingegen eine spekulative Frage, die keine kohärente Antwort zulässt. Wir sollten jedoch bereit sein, Teilbereiche zu verteidigen, in denen sich Fortschritt ereignet hat, und uns erinnern, dass dies nie ohne Verlust, Tragödie und Konflikt geschehen ist. Ich danke Peter Gordon für seine Hartnäckigkeit mir gegenüber in diesem Punkt. Und eine der wichtigsten Aufgaben, die in unserem aktuellen geschichtlichen Moment vor uns liegen, ist es, die Errungenschaften der modernen Wissenschaft 
Wenn wir über Geltung reden möchten, müssen wir einander als Wesen respektieren, die wir mit guten Gründen zu überzeugen versuchen, wie ich, in Übereinstimmung mit Habermas, anderswo dargelegt habe. ${ }^{66}$ Solche Dialoge haben Verfahrensbedingungen wie Gleichheit und Reziprozität unter Dialogpartnern beim Einbringen von Themen, beim Hinterfragen von Sprachregeln und selbst im reflexiven Infragestellen der dialogischen Versuchsanordnung. Diese Diskursregeln sind in den Rechtsstaat, parlamentarische Geschäftsordnungen und die öffentlichen Sphären von Verfassungsdemokratien eingegangen. Sie sind nicht unfehlbar und sie müssen herausgefordert und revidiert werden, aber ohne die Universalien, die in diese institutionellen Praktiken eingelassen sind, die wir von der politischen Moderne ererbt haben, können wir Fragen von Ausbeutung, Ungerechtigkeit, Ausgrenzung, Zum-Schweigen-Bringen und von vielem anderem nicht beantworten. Habermas hat uns mit großer Detailversessenheit und unübertrefflicher Belesenheit gezeigt, wie diese Geltungsnormen aus der Begegnung von Glauben und Wissen im Westen hervorgegangen sind. Meine Behauptung ist es, dass diese Normen reicher und nuancierter hätten ausfallen können, hätte man den ,affektiven Geschichten“67 der Stimmen der Ausgeschlossenen mehr Gehör geschenkt. ${ }^{68}$

Aus dem Englischen von Matthias Warkus

\section{Literatur}

Allen, A. (2016), The End of Progress: Decolonizing the Normative Foundations of Critical Theory, New York.

Anghie, A. (2005), Imperialism, Sovereignty and the Making of International Law, Cambridge. Arendt, H. (1967), Vita activa [1958], München u. Berlin.

mit einem Verständnis der Zukunft des Planeten in einer Weise zu verbinden, dass wissenschaftliche Technologie nicht die Dienstmagd einer ausbeuterischen kapitalistischen oder staatskapitalistischen Ökonomie bleibt.

66 Vgl. Benhabib (2002).

67 Chakrabarty (2008).

68 Danksagung: Ich möchte Stefan Eich, Peter Gordon, Dick Howard, Thomas McCarthy, Nishin Nathwani und Joel Withebook für ihre Kommentare und Kritik zu früheren Entwürfen dieses Aufsatzes danken, außerdem James Sleeper für minutiöse stilistische wie auch inhaltliche Kommentare. Vielen Dank auch an Peter Verovšek, der bei der Tagung der American Political Science Association am 12.9.2020 eine Sitzung zu Habermas' Buch organisierte und mich dazu drängte, mich damit auseinanderzusetzen. 
Baier, A. (1987), Hume, the women's moral theorist?, in: Kittay, E. F., u. Meyers, D. T. (Hg.), Women and Moral Theory, Totowa, N. J., 37-55.

Baier, A. (1993), Hume: The reflective women's epistemologist?, in: Antony, L. (Hg.), A Mind of One's Own: Feminist Essays on Reason and Objectivity, Boulder, Colo., 38-52.

Barber, B. (1996), Coca-Cola und Heiliger Krieg, München.

Baynes, K. (1991), The Normative Grounds of Social Criticism, Albany, N. Y.

Benhabib, S. (1986), Critique, Norm and Utopia: A Study of the Foundations of Critical Theory, New York; dt.: dies. (2017), Kritik, Norm und Utopie. Die normativen Grundlagen der Kritischen Theorie, übers. v. Kohlhaas, P., Frankfurt am Main.

Benhabib, S. (1995), Der verallgemeinerte und der konkrete Andere, in: Selbst im Kontext, Frankfurt am Main, 161-191.

Benhabib, S. (2002), The Claims of Culture: Equality and Diversity in the Global Era, Princeton, N. J. Benhabib, S. (2019), What Is Living and What Is Dead in John Rawls's Theory of Justice?, in: The Nation (11./18.11.2019), 28-33.

Benhabib, S., Butler, J., Fraser, N., u. Cornell, D. (1993), Der Streit um Differenz, Frankfurt am Main.

Benhabib, S., Butler, J., Fraser, N., u. Cornell, D. (1996), Feminist Contentions: A Philosophical Exchange, London u. New York.

Benhabib, S., u. Cornell, D. (Hg.) (1987), Feminism as Critique: Essays on the Politics of Gender in Late-Capitalist Societies, Minneapolis.

Benhabib, S., u. Nicholson, L. (1987), Politische Philosophie und die Frauenfrage, in: Fetscher, I., u. Münkler, H. (Hg.), Handbuch der politischen Ideen 5, München, 513-562.

Benjamin, W. (1992), Über den Begriff der Geschichte [1940], in: Sprache und Geschichte, Stuttgart, 141-155.

Bloch, B. (2020), The Unfinished Project of Enlightenment, in: Boston Review (18.6.2020).

Brandom, R. (1994), Making It Explicit: Reasoning, Representing, and Discursive Commitment, Cambridge, Mass.

Buck-Morss, S. (2009), Hegel, Haiti, and Universal History, Pittsburgh, Pa.

Chakrabarty, D. (2008), Provincializing Europe: Postcolonial Thought and Historical Difference [2000], Princeton, N. J. [hier ad hoc übers. v. Warkus, M.].

Cornell, D. (1999), Beyond Accommodation: Ethical Feminism, Deconstruction, and the Law [1991], London u. New York.

Daut, M. (2020), When France extorted Haiti - the greatest heist in history, in: The Conversation (30.6.2020), URL: https://theconversation.com/when-france-extorted-haiti-the-greatestheist-in-history-137949 (11.6.2021).

Dewey, J. (2008), The Quest for Certainty, in: The Later Works of John Dewey (1925-1953): Vol. 4 (1929) [1984], hg. v. Boydston, J. A., Carbondale, Ill.

Eisenstadt, S. N. (2000), Multiple Modernities, in: Daedalus 129.1, 1-29.

Eisenstadt, S. N. (Hg.) (2002), Multiple Modernities, Piscataway, N. J.

Eisenstadt, S. N. (2003), Comparative Civilizations and Multiple Modernities, Leiden u. a.

Elshtain, J. B. (1981), Public Man, Private Woman, Princeton, N. J.

Finlayson, J. G. (2019), The Habermas-Rawls Debate, New York.

Finlayson, J. G., u. Freyenhagen, F. (Hg.) (2011), Habermas and Rawls: Disputing the Political, London u. New York.

Forst, R. (1996), Kontexte der Gerechtigkeit, Frankfurt am Main.

Forst, R. (2021), The autonomy of autonomy: On Jürgen Habermas's Auch eine Geschichte der Philosophie, in: Constellations 28.1, 17-24. 
Fraser, N. (1989), Unruly Practices: Power, Discourse, and Gender in Contemporary Social Theory, Minneapolis.

Fraser, N. (1997), Justice Interruptus: Critical Reflections on the „Postsocialist“ Condition, London u. New York.

Fraser, N., u. Honneth, A. (2003). Redistribution of Recognition. A Philosophical Exchange, London u. New York.

Fraser, N., u. Jaeggi, R. (2018), Capitalism: A Conversation in Critical Theory, Cambridge.

Gališanka, A. (2019), John Rawls: The Path to a Theory of Justice, Cambridge, Mass.

Gordon, P. E. (2021), Is there an asymmetry problem in the genealogy of postmetaphysical reason?, in: Constellations 28.1, 45-50.

Habermas, J. (1968), Arbeit und Interaktion. Bemerkungen zu Hegels Jenenser ,Philosophie des Geistes“ [1967], in: Technik und Wissenschaft als ,Ideologie‘, Frankfurt am Main, 9-47.

Habermas, J. (1978), Theorie und Praxis [1963], Frankfurt am Main.

Habermas, J. (1979), Consciousness-Raising or Redemptive Criticism: The Contemporaneity of Walter Benjamin, in: New German Critique 17 (Special Walter Benjamin Issue), 30-59.

Habermas, J. (1992), Faktizität und Geltung, Frankfurt am Main.

Habermas, J. (1995a), Theorie des kommunikativen Handelns 1. Handlungsrationalität und gesellschaftliche Rationalisierung [1981], Frankfurt am Main.

Habermas, J. (1995b), Theorie des kommunikativen Handelns 2. Zur Kritik der funktionalistischen Vernunft [1981], Frankfurt am Main.

Habermas, J. (2001), Die Zukunft der menschlichen Natur, Frankfurt am Main.

Habermas, J. (2019a), Auch eine Geschichte der Philosophie 1, Berlin.

Habermas, J. (2019b), Auch eine Geschichte der Philosophie 2, Berlin.

Hegel, G. W. F. (1986), Phänomenologie des Geistes [1807] (= Werke 3), Frankfurt am Main.

Herder, J. G. (2020), Auch eine Philosophie der Geschichte zur Bildung der Menschheit [1774], Berlin.

Kant, I. (2019), Idee zu einer allgemeinen Geschichte in weltbürgerlicher Absicht [1784], Göttingen.

Lloyd, G. (2004), Man of Reason: „Male“ and „Female“ in Western Philosophy [1984], London u. New York.

Marx, K. (1960), Der achtzehnte Brumaire des Louis Bonaparte [1852], in: ders. u. Engels, F., Marx-Engels-Werke 8, Berlin, 111-207.

McCarthy, T. A. (1994), Kantian Constructivism and Reconstructivism: Rawls and Habermas in Dialogue, in: Ethics 105.1, 44-63.

McCarthy, T. A. (2015), Rassismus, Imperialismus und die Vorstellung menschlicher Entwicklung [2009], Berlin [E-Book, ohne Paginierung zitiert].

Nussbaum, M. (1999), Sex and Social Justice, Oxford.

Okin, S. M. (1979), Women in Western Political Thought, Princeton, N. J.

Okin, S. M. (1991), Justice, Gender and the Family, New York.

Pateman, C. (1988), The Sexual Contract, Stanford, Calif.

Pinkard, T. (1996), Hegel's Phenomenology of Spirit. The Sociality of Reason, Cambridge.

Pinkard, T. (2000), Hegel: A Biography, Cambridge.

Pippin, R. B. (2008), Hegel's Practical Philosophy, Cambridge.

Rawls, J. (1995), Reply to Habermas, in: The Journal of Philosophy 92.3, 132-180.

Rawls, J. (2009), A Brief Inquiry into the Meaning of Sin and Faith: With „On my Religion“, hg. v. Nagel, T., Cambridge, Mass.

Ricœur, P. (1993), Die Interpretation. Ein Versuch über Freud [1969], 4. Aufl., Frankfurt am Main. 
Rorty, R. (2009), Philosophy and the Mirror of Nature (30th anniversary ed.) [1979], Princeton, N. J.

Rousseau, J.-J. (2019), Diskurs über die Ungleichheit [1755], 7. Aufl., Paderborn.

Sellars, W. (1997), Empiricism and the Philosophy of Mind [1956], Cambridge, Mass.

Taylor, C. (1972), The Opening Arguments of the Phenomenology, in: Maclntyre, A. (Hg.), Hegel: A Collection of Critical Essays, New York, 151-188.

Taylor, C. (2001), Two Theories of Modernity, in: Gaonkar, D. (Hg.), Alternative Modernities, Durham, N. C., 172-196.

Weber, M. (1994), Wissenschaft als Beruf [1917/1919], in: Wissenschaft als Beruf. Politik als Beruf (=MWS I/17), Tübingen, 1-23.

Weber, M. (2010), Die protestantische Ethik und der Geist des Kapitalismus [1920], 3. Aufl., München.

Williams, B. (1984), Moralischer Zufall [1981], Königstein im Taunus.

Yovel, Y. (2009), The Other Within: The Marranos: Split Identity and Emerging Modernity, Princeton, N. J. 